Running Head: ERROR MONITORING AND BRAIN DEVELOPMENT

\title{
The interplay of age and pedagogy in maturation of error-monitoring
}

Solange Denervaud ${ }^{1,2}$, David A. Tovar ${ }^{3}$, Jean-François Knebel ${ }^{1,2}$, Emeline Mullier ${ }^{4}$, Yasser AlemánGómez ${ }^{4,5}$, Patric Hagmann ${ }^{4 *}$ \& Micah M. Murray ${ }^{1,2,3,6^{*}}$

(1)

${ }^{1}$ The Laboratory for Investigative Neurophysiology (The LINE), Department of Radiology, Lausanne University Hospital and University of Lausanne, 1011 Lausanne, Switzerland

${ }^{2} \mathrm{CIBM}$ Center for Biomedical Imaging, Lausanne, Switzerland

${ }^{3}$ Vanderbilt Brain Institute, Vanderbilt University, Nashville, TN, USA

${ }^{4}$ Connectomics Lab, Department of Radiology, Lausanne University Hospital and University of Lausanne, 1011 Lausanne, Switzerland

${ }^{5}$ Center for Psychiatric Neuroscience, Department of Psychiatry, Lausanne University Hospital and University of Lausanne, Prilly, Switzerland.

${ }^{6}$ Department of Ophthalmology, Fondation Asile des aveugles and University of Lausanne, Switzerland

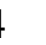


Running Head: ERROR MONITORING AND BRAIN DEVELOPMENT

2 Error monitoring allows us to adapt to an inherently dynamic environment, and relies mainly

3 on the anterior cingulate cortex (ACC). Its protracted maturation suggests a window for

4 experience-dependent plasticity. To investigate this possibility, we measured error-related

5 response-locked potentials components with morphometric magnetic resonance imaging (MRI)

6 measures of the ACC in twenty-six schoolchildren, aged 6-12 years, enrolled in either

7 a Montessori or a traditional curriculum. We show that the caudal ACC undergoes significant

8 morphometric changes during this age range that relate to error detection ERP activity.

9 Furthermore, pedagogy modulates source localization activity related to error detection within

10 the caudal ACC amongst others. We provide early evidence for a window of opportunity

11 regarding the acquisition of error monitoring, with implications for education.

12

13 Keywords: Self-monitoring - error monitoring - brain development - anterior cingulate cortex

14 - experience-dependent plasticity - school pedagogy 
Running Head: ERROR MONITORING AND BRAIN DEVELOPMENT

The interplay of age and pedagogy in maturation of error-monitoring

2 To function well in contemporary society, children need to gain independence and flexibility.

3 Central to these adaptive processes stands the error monitoring system, which detects

4 unexpected outcomes and provides opportunity to swiftly adjust behaviors (Ullsperger,

5 Danielmeier, \& Jocham, 2014). Error monitoring strongly relies on the caudal subregion of

6 the anterior cingulate cortex (cACC), a central hub of the salience network (Margulies et al.,

7 2007). Functional maturation of the cACC mainly occurs from 6 to 12 years of age (Kelly et

8 al., 2009). This maturation window suggests a possible sensitive in which incorrect responses

9 are learned through experience-dependent plasticity. To address this possibility, the present

10 study investigated the relationship between cortical thickness changes within ACC subregions

11 and established error-related electroencephalographic (EEG) components in 6-12 year-old

12 children experiencing different error monitoring styles at school.

13 Our routine behaviors rely on automatized actions with expected outcomes. However,

14 these expectations are occasionally unmet and generate a mismatch between planned and

15 actual outcomes, such as flipping a light switch without the light coming on. In these

16 circumstances, the error monitoring system is triggered, signaling the type of adaptation

17 required to compensate for the unexpected event (e.g., adversity, conflict, incorrect response).

18 An optimal error monitoring system provides potential explanations: "Maybe the bulb needs

19 changing”, and can facilitate putting them into action (Ullsperger et al., 2014).

There is no direct measure of error monitoring, but indirect informative markers have

21 been widely studied and reported in the context of incorrect responses. At a behavioral level,

22 studies have generally focused on reaction times (RTs), which are usually slower after

23 incorrect compared to correct responses (referred to as post-error slowing; Notebaert et al.,

24 2009). There is also a long history of EEG studies examining the time course of events

25 following incorrect responses. Despite differences in the protocols used, as long as speed and 


\section{Running Head: ERROR MONITORING AND BRAIN DEVELOPMENT}

1 accuracy are emphasized, a similar sequence of brain components time-locked to the

2 behavioral response has been reported in adults (Ullsperger et al., 2014). The first is an early

3 frontocentral negativity (typically peaking 50-100ms post-response onset), dubbed the error-

4 related negativity (ERN; Ullsperger \& Danielmeier, 2016). The second is a later and slower

5 response ( $200-400 \mathrm{~ms}$ post-response onset) with a more central scalp distribution, called the

6 error positivity $(\mathrm{Pe})$. They are thought to reflect an early task-unspecific detection of need for

7 adjustments (ERN) and a later task-specific selective attention for orientation and learning

8 (Pe) or conscious evaluation (Ullsperger \& Danielmeier, 2016). ERN and Pe together reflect a

9 built-in error-detection system (Elton, Band, \& Falkenstein, 2000) of respectively, low-level

10 perceptual ability for fast detection of mismatch (Falkenstein, Hohnsbein, Hoormann, \&

11 Blanke, 1991; Murphy, van Moort, \& Nieuwenhuis, 2016) and for later top-down processes

12 of adaptation/evaluation (Falkenstein, 2000; Nieuwenhuis, Ridderinkhof, Blom, Band, \&

13 Kok, 2001; Overbeek, Nieuwenhuis, \& Ridderinkhof, 2005). While these markers are well-

14 studied in adults, developmental data are currently more scarce and inconsistent.

Both components have been observed in preschoolers (Brooker, Buss, \& Dennis, 2011).

16 For example, the ERN was recorded in children as young as 3-4 years old (Grammer,

17 Carrasco, Gehring, \& Morrison, 2014; Smulders, Soetens, \& van der Molen, 2016). The capacity to detect behavioral errors seems to gain in efficiency with age. Indeed, many of the pediatric studies on ERN report an increase in amplitude with age, reaching adult-like levels at late adolescence (Tamnes, Walhovd, Torstveit, Sells, \& Fjell, 2013). However, there is also

21 evidence that the trajectory of this maturation is non-linear and inconsistent across ERN and

22 Pe components (Tamnes et al., 2013). For example, there are reports of reduced ERN

23 amplitude during adolescence (between 10-13 y.o.), despite constant Pe amplitude. Such

24 fluctuations are thought to reflect protracted maturation of error monitoring within the 


\section{Running Head: ERROR MONITORING AND BRAIN DEVELOPMENT}

1 underlying brain structures, i.e., the $\mathrm{cACC}$, and may be related to experience-dependent

2 plasticity.

ACC maturation follows a caudal to rostral path, from birth to late adolescence, tightly related to the successive gain in self-monitoring abilities at the motor, cognitive and socialaffective levels (Margulies et al., 2007; Posner \& Rothbart, 2007; Posner, Rothbart, Sheese, \& Tang, 2007). Error monitoring relies on the cACC. This region readily captures mismatches and in turn activates other networks to adapt behavior (e.g., medial frontal cortex; see Danielmeier, Eichele, Forstmann, Tittgemeyer, \& Ullsperger, 2011). The activity of the cACC undergoes significant changes during childhood from around 6 to 12 years of age (Kelly et al., 2009; Velanova, Wheeler, \& Luna, 2008), raising the possibility that during

11 these years, children learn to monitor themselves when facing unexpected events at the cognitive level, such as incorrect responses. Error monitoring development may thus be subject to environmental influences, through experience-dependent plasticity, such as learning strategies reinforced within schooling pedagogies. responses. Traditional pedagogy primarily involves teacher-led curricula, where knowledge is evaluated through tests with quantitative assessments (i.e., grades), delayed feedback, as well as within an environment of peers from the same age. Instead, child-centered-constructivist pedagogies, such as the Montessori pedagogy, encourages a children-led trial-and-error approach to learning, where knowledge is self-evaluated or receives qualitative feedback,

21 without grades, in an environment of peers mixed across different ages (Lillard, 2005;

22 Marshall, 2017; Montessori, 1936). These different pedagogical approaches were recently reported to impact error monitoring abilities at the behavioral and neural level in Montessori 


\section{Running Head: ERROR MONITORING AND BRAIN DEVELOPMENT}

1 Denervaud, Hess, Sander, \& Pourtois, 2020; Denervaud, Knebel, Immordino-Yang, \&

2 Hagmann, 2020).

3 So far, developmental studies focused on error monitoring characterization (i.e. specific

4 features, etc.) or individual trait or state differences in healthy or clinical populations (anxiety,

5 mindsets, obsessive-compulsive disorders, etc.). Inconsistencies in reported outcomes

6 preclude establishing a clear developmental trajectory. Here, we evaluated cACC maturation

7 through cortical thickness changes, brain components for incorrect versus correct responses

8 (ERN and $\mathrm{Pe}$ ) changes with age, and the extent to which pedagogy experienced at school

9 modulates children's error monitoring biomarkers between 6 and 12 years of age.

Specifically, we hypothesized that (i) ACC maturation (i.e., cortical thinning; Gogtay et

11 al., 2004) would occur mainly within its caudal subregion, based on previous studies (Kelly et

12 al., 2009; Velanova et al., 2008); (ii) ERN would show changes with age and would relate to

13 cACC cortical thinning (maturation of the error monitoring system), while not Pe; and (iii)

14 experience-dependent plasticity would be observed according to the pedagogical experiences

15 of the children. We expected to observe enhanced error monitoring biomarkers in Montessori-

16 schooled children, compared to the traditionally-schooled children. Previous comparative

17 studies (Montessori- versus traditionally-schooled children) reported younger error-detection

18 and self-correction (Denervaud, Knebel, et al., 2020), greater and faster engagement with

19 errors originating within the ACC (Denervaud et al., 2020), and better academic achievement

20 (Denervaud, Knebel, Hagmann, \& Gentaz, 2019), which may translate into stronger brain

21 responses (Hirsh \& Inzlicht, 2010).

\section{Methods}

24 The experiment was conducted in accordance with the Declaration of Helsinki. The 25 experimental procedures were approved by University ethical committee. 
Running Head: ERROR MONITORING AND BRAIN DEVELOPMENT

\section{Participants}

Written parental consent was obtained for each child, and informed assent was provided by each participant. No child had a history of a neurological or psychiatric illness. At each session, children received a voucher of $\sim 35$ USD. In total, 31 children completed the experiment as part of a larger study, including neuroimaging and behavioral measures aimed at evaluating the impact of school environment on development. Selection criteria were age (5 to 14 y.o.) and schooling system (children had to be enrolled in a Montessori or traditional school system). Children with an error rate lower than $10 \%(\mathrm{~N}=3)$, technical difficulties with EEG recording $(\mathrm{N}=1)$, or dental brace interference with MRI recording $(\mathrm{N}=1)$ were removed from the study, leaving 26 children for the analyses (mean age $\pm \mathrm{SD}=8.9 \pm 1.6$ years; all righthanded with normal or corrected-to-normal vision). Of these 26 children, all participated in the aforementioned behavioral study on performance-monitoring (Denervaud, Knebel, et al., 2020), and 12 of them participated in the aforementioned error monitoring fMRI study (Denervaud et al., 2020). Thus, all of the EEG and anatomical MRI analyses are reported here for the first time.

To explore the idea of experience-dependent plasticity through pedagogy, we accounted for the Montessori or traditional schooling background of the child. To control, as best as possible, for the selection bias of Montessori-schooled children from private school and children from traditional public schools, we collected information about children's fluid intelligence (Raven, Raven, \& Court, 2003), trait anxiety (STAI-Y2; Spielberger \& Vagg, 1984) and mindsets (implicit theories of intelligence; Blackwell, Trzesniewski, \& Dweck, 2007). We additionally collected information about parental socio-economic status (Genoud, 2011), education style and environment at home through a tailor-made questionnaire. Details 
1 of each questionnaire are provided in the supplementary materials. Demographic data were

2 collected online post-recording.

\section{MRI acquisition and processing}

Structural imaging was collected at the $\mathrm{X}$, on a Siemens 3T Prisma-Fit MR scanner, with a 64-channel head-coil. For each child, a 3-dimensional high-resolution isotropic T1-weighted sequence (MPRAGE) was acquired $(\mathrm{TR}=2000 \mathrm{~ms}, \mathrm{TE}=2.47$ ms, 208 slices; voxel size $=1 \mathrm{~mm}^{3}$, flip angle $=8^{\circ}$, percent phase $\mathrm{FOV}=100$ ). Individual T1-weighted images were processed using the FreeSurfer 6.0.0 software (http://surfer.nmr.mgh.harvard.edu), a widely-used open-source image processing software package. FreeSurfer's image processing steps include brain extraction, automated Talaraich transformation, subcortical structures parcellation and brain tissue segmentation into white and grey matter.

Once white matter, grey matter and cerebrospinal fluid volumes were generated, the white (grey/white interface) and grey (csf/grey interface) cortical surfaces were reconstructed with the methods described by Fischl and colleagues (Fischl et al., 2002; Fischl, Sereno, \& Dale, 1999). Cortical parcellation in gyral-based ROIs were calculated according to the Desikan-Killiany cortical atlas (Desikan et al., 2006), which contains 34 cortical structures for each hemisphere. Mean cortical thickness for each brain region was calculated as the average distance between the cortical surfaces for all vertices belonging to that region.

The cortical thickness, which represents a direct measure of the amount of grey matter contained in cortical layers along the perpendicular direction to each point on the surface, was used as a brain maturation biomarker (Gogtay et al., 2004). Based on our a priori hypothesis, the rostral ACC, cACC, and posterior cingulate cortex (PCC) were selected as regions of interest (ROIs) for the further analyses.

\section{EEG procedure and processing}




\section{Running Head: ERROR MONITORING AND BRAIN DEVELOPMENT}

1 To explore the effect of error monitoring in school-like procedures, children performed a

2 speeded continuous recognition task, modified for children from Thelen et al. (2014). In this

3 task, children were asked to press a key as fast and accurately as possible, differentiating

4 initial (i.e., 'new') versus repeated (i.e., 'old') presentations of line drawings (see Figure S1).

$5 \quad$ Initial and repeated presentations of an item were pseudo-randomized within a block of 160

6 trials. Each object was repeated only once within each experimental block (i.e. 80 drawings

7 for 160 trials). Children performed four blocks (640 trials in total). The line drawings were

8 taken from a standardized set (Snodgrass \& Vanderwart, 1980) or obtained from an online

9 library (dgl.microsoft.com). The experiment took place in a sound-attenuated chamber (MDL

10 102126E from Whisperroom Inc.), where children were seated centrally in front of a 20" LCD

11 computer monitor that was located about $80 \mathrm{~cm}$ away from them to produce a visual angle of

$12 \sim 4^{\circ}$ for the line drawings. The task was designed and controlled by PsychoPy 3.0 (Peirce et

13 al., 2019), and all behavioural data were recorded in conjunction with a serial response box

14 (Psychology Software Tools, Inc.; www.pstnet.com).

15 Continuous EEG was acquired at $1024 \mathrm{~Hz}$ through a 64-channel Biosemi ActiveTwo

16 AD-box (http://www.biosemi.com) referenced to the common mode sense (CMS; active

17 electrode) and grounded to the driven right leg (DRL; passive electrode), which functions as a

18 feedback loop driving the average potential across the electrode montage to the amplifier

19 zero.

Pre-processing and analyses were performed using both homemade python scripts

21 using Anaconda distribution (Python Software Foundation. Python Language Reference,

22 version 3.5); Matlab (Mathworks, Natick, MA, USA Version 7.13); and the Cartool freeware

23 (Brunet et al. 2011). Data were first filtered with a $2^{\text {nd }}$ order Butterworth filter (12dB/octave

24 roll-off; $1.0 \mathrm{~Hz}$ high-pass; $60 \mathrm{~Hz}$ low-pass; $50 \mathrm{~Hz}$ notch). The filters were computed linearly in

25 both forward and backward directions to eliminate phase shifts. Then EEG epochs were time- 


\section{Running Head: ERROR MONITORING AND BRAIN DEVELOPMENT}

1 locked to the motor response and spanned $200 \mathrm{~ms}$ pre-response and $500 \mathrm{~ms}$ post-

2 response. Epochs with amplitude deviations in excess of $\pm 100 \mu \mathrm{V}$ at any channel,

3 except for those labeled as 'bad' due to poor electrode-skin contact or damage, were

4 considered artifacts and were excluded. Data from 'bad' channels (mean electrodes

$5 \pm \mathrm{SD}=2.04 \pm 2.49$ channels) were interpolated using 3D splines (Perrin et al., 1987).

6 Prior to group-averaging response-locked potentials were baseline-corrected (-200 to -

$7 \quad 100 \mathrm{~ms}$ from the response).

Two error-related components were extracted: the ERN and the Pe. To extract these components a peak-to-peak analysis was performed between the pre-motor response periods (100 to $0 \mathrm{~ms}$ ) and periods of interest of each component (ERN: 0-100ms and Pe: 250-450ms).

11 Based on previous literature (e.g.Aarts, De Houwer, \& Pourtois, 2013; Grammer et al., 2014; Meyer, Weinberg, Klein, \& Hajcak, 2012) and confirmed through an exploratory searchlight decoding analysis (Etzel, Zacks, \& Braver, 2013) (see Figure S2), we selected scalp locations ( $\mathrm{FCz}$ and $\mathrm{Cz})$ and the time period of interest $(0-100 \mathrm{~ms}$ and $250-450 \mathrm{~ms})$, respectively, for the ERN and Pe components.

\section{Statistical Analyses}

17 Statistical analyses were computed using R Computer Software.

\section{Group variables} difference were run on the different control variable scores to statistically determine significant differences between the two groups of children (experiencing Montessori versus traditional pedagogy), with a false-rate discovery (FDR) $p$-value correction at $\mathrm{q}=0.05$. 
To investigate the maturation of the ACC cortical thickness, a multiple analysis of

2 covariance (MANCOVA) was run on cortical thickness measures of the three ROIs as

3 dependent variables: rostral ACC, caudal ACC, and PCC, with age as a covariate and group

4 (experiencing Montessori versus traditional pedagogy) as a factor (with $\alpha<0.05$ ). Given the

5 effect of gender on cortical thickness (Gennatas et al., 2017), we included gender as a factor

6 and its interaction with group.

\section{Effect of Age and Pedagogy on ERN and Pe}

First, independent t-tests were computed on accuracy and error rates, to statistically evaluate differences between Montessori and traditionally-schooled children. Second, a repeated-measures analysis of covariance (rmANCOVA) with response type (correct, incorrect) as the within-subject factor, pedagogy (Montessori, traditional) as the betweensubjects factor, and age as a covariate, was run on reaction times (RTs) (with $\alpha<0.05$ ). Finally, to investigate the effect of age and pedagogy on ERN and Pe, rmANOVAs were conducted on the peak-to-peak data with response type (correct, incorrect) as the withinsubjects factor, pedagogy (Montessori, traditional) as the between-subjects factor and age as a covariate (with $\alpha<0.05$ ). Post-hoc Tukey tests were performed when appropriate.

\section{Relation between ACC cortical thickness, ERN and Pe}

We computed the difference between incorrect and correct peak-to-peak values for each error-related brain component (i.e., $\triangle \mathrm{ERN}$ and $\Delta \mathrm{Pe}$ ). Multiple linear regression analyses were calculated to predict $\triangle \mathrm{ERN}$ and $\Delta \mathrm{Pe}$ based on the cortical thickness measures of the rostral ACC, the $\mathrm{ACC}$ and the $\mathrm{PCC}$, the age, and the pedagogy.

Additionally, we applied the local auto-regressive average distributed linear inverse solution (LAURA; Grave de Peralta Menendez, Gonzalez Andino, Lantz, Michel, \& Landis, 2001) to an average period around $\pm 10 \mathrm{~ms}$ around the ERN response-locked peaks. We visualize and statistically contrast the likely underlying sources with use of the software brain 


\section{Running Head: ERROR MONITORING AND BRAIN DEVELOPMENT}

1 template for 7.5-13.5 year-old children (provided by Cartool). Statistical analysis entailed the

2 same mixed model design as above and was performed using STEN software (Knebel \&

3 Notter, 2012). A spatial extent criterion of at least 5 contiguous significant nodes $(p<0.05)$

4 was applied. F-maps thresholds by significant points were displayed, after separating

5 distinguished blobs.

\section{Results}

\section{Group variables}

The groups were comparable in terms of age, gender, fluid intelligence, trait anxiety, and mindsets (all $p>0.05$ ). Furthermore, parental SES, education style and environment at home were similar as well (Table 1).

\section{Effect of Age and Pedagogy on ACC cortical thickness}

Corroborating our hypothesis, the thickness of the cACC significantly decreased with age ( $p=0.047$, Figure 1A), but not within the PCC $(p=0.292)$, or the $\operatorname{rACC}(p=0.186)$. There was an effect of pedagogy on PCC cortical thickness $(p=0.018)$, with the traditionallyschooled children showing thinner PCC (mean thickness $\pm \mathrm{SD}=5.51 \pm 0.12 \mathrm{~mm}$ ) compared to the Montessori-schooled children (mean thickness $\pm \mathrm{SD}=5.75 \pm 0.33 \mathrm{~mm}$ ). There was no gender-specific cortical thickness differences for the ROIs $(p>0.208)$.

\section{Effect of Age and Pedagogy on ERN and Pe}

Overall, participants committed an average of $17.3 \%(\mathrm{SD}=11.4 \%)$ errors, with an accuracy rate of $73.2 \%(\mathrm{SD}=19.7 \%)$. Age was unrelated to accuracy, $\mathrm{r}_{(26)}=-0.018, p=0.93$. Both error and accuracy rates were comparable between the Montessori and traditionallyschooled children (respectively $\mathrm{t}_{(24)}=0.81, p=0.43$, Cohen's $\mathrm{d}=0.32$ and $\mathrm{t}_{(24)}=0.25, p=0.80$, Cohen's $\mathrm{d}=0.10$ ). As shown in previous studies, participants were faster on incorrect than correct trials $\left(\mathrm{M}_{\mathrm{RT}} \pm \mathrm{SD}=915 \pm 357 \mathrm{~ms}\right.$ versus $\left.1050 \pm 442 \mathrm{~ms} ; \mathrm{F}_{(1,23)}=6.37, p=0.019, \eta_{\mathrm{p}}{ }^{2}=0.22\right)$. Furthermore, age had a significant effect on RT of both correct and incorrect trials 
$1 \quad\left(\mathrm{~F}_{(1,23)}=19.74, p<.001, \eta_{\mathrm{p}}{ }^{2}=0.46\right)$, with older children being faster. There was no evidence of a

2 significant effect of pedagogy $(p=0.84)$ nor of an interaction between pedagogy and RT

$3(p=0.64)$. We obtained comparable behavioral outcomes in both groups.

For both groups of children, ERN peak-to-peak amplitude was significantly more negative on incorrect than correct trials $\left(-5.18 \pm 2.95 \mu \mathrm{V}\right.$ versus $-4.16 \pm 3.12 \mu \mathrm{V} ; \mathrm{F}_{(1,23)}=6.69$, $p=0.016, \eta_{\mathrm{p}}{ }^{2}=0.23$, Figure 1B). There was a main effect of Pedagogy as well $\left(\mathrm{F}_{(1,23)}=5.34\right.$, $\left.p=0.030, \eta_{\mathrm{p}}{ }^{2}=0.19\right)$. Montessori-schooled children had overall more negative brain responses than traditionally-schooled children $\left(p_{\text {tukey }}=0.031\right)$. There was a significant interaction of Response Type (correct, incorrect) on Age, $\mathrm{F}_{(1,23)}=5.39, p=0.030, \eta_{\mathrm{p}}{ }^{2}=0.19$, with increased negativity for incorrect responses, while increased positivity for correct responses in younger children. The Pedagogy $\times$ Response Type interaction was not significant $(p>0.05)$.

Analysis of the Pe peak-to-peak amplitude revealed a significant main effect of Response Type (correct, incorrect; $\mathrm{F}_{(1,23)}=9.55, p=0.005, \eta_{\mathrm{p}}{ }^{2}=0.29$, Figure $\mathrm{S} 3$ ). There was also a significant interaction of Response Type (correct, incorrect) on Age, $\mathrm{F}_{(1,23)}=5.74, p=0.025$, $\eta_{\mathrm{p}}{ }^{2}=0.20$, with increasing age related to higher positivity for correct responses, but not for incorrect responses.

Relation between ACC cortical thickness, $\triangle E R N$ and $\triangle P e$

Children's $\triangle \mathrm{ERN}$ was reliably predicted by Age $\left(\mathrm{F}_{(1,20)}=8.77, p=0.008\right)$ and cACC cortical thickness $\left(\mathrm{F}_{(1,20)}=5.05, p=0.036\right)$ (Figure 1C). Larger $\Delta \mathrm{ERN}$ were related to thicker cortical values. Neither pedagogy, nor PCC or rACC were predictors of $\triangle \mathrm{ERN}(p=0.166)$.

Children's $\Delta \mathrm{Pe}$ was reliably predicted by Age only $\left(\mathrm{F}_{(1,20)}=4.36, p=0.050\right)$, with older children showing smaller $\Delta \mathrm{Pe}$. No other factor was found to be a significant predictor of $\Delta \mathrm{Pe}$ $(p>0.517)$.

For the ERN, statistical analyses of distributed source estimations revealed significant interactions between Response Type and Pedagogy; within the left cACC and mPFC, and 


\section{Running Head: ERROR MONITORING AND BRAIN DEVELOPMENT}

1 within the left inferior temporal cortex and cerebellum (Figure 1D). Post-hoc t-tests revealed

2 higher ERN amplitudes for correct responses versus incorrect responses for Montessori versus 3 traditionally-schooled children.

\section{Discussion}

Error monitoring is central to flexible adaptation, and mainly relies on the ACC. The current study investigated the extent to which cortical thickness changes within subregions of the ACC were related to the EEG components of error monitoring (i.e., $\Delta \mathrm{ERN}$ and $\Delta \mathrm{Pe}$ ), and whether experience-dependent plasticity through pedagogy (i.e., interaction) was observable.

Regarding the morphometric data, we report brain maturation (cortical thinning) within the cACC that corroborates previous work showing functional changes from around 6 to 12 years of age (Kelly et al., 2009; Velanova et al., 2008). The cACC, together with bilateral insula, form the salience network (Menon \& Uddin, 2010), which changes during these years (Uddin, Supekar, Ryali, \& Menon, 2011).

The salience network is triggered for "oddball" events, such as incorrect responses. It may be that children at that age are more sensitive to cognitive mismatches, optimizing their error monitoring development plasticity. This attentional bias may contribute to both early perceptive abilities, as reflected by the ERN peak, as well as in feedback integration for adaptive processes, contributing to the observed Pe peak. We also found thicker PCC in Montessori compared to traditionally-schooled children. PCC is a central hub of the default mode network, often reported to be involved in internally-directed and creative cognition (Beaty et al., 2014; Immordino-Yang, Christodoulou, \& Singh, 2012; Leech \& Sharp, 2014; Raichle et al., 2001). Previous work has shown an effect of Montessori pedagogy on creative abilities and mindful thinking (Denervaud et al., 2019; Lillard \& Else-Quest, 2006; Lillard, 2011; Rathunde, 
1 2001). Future work will be necessary to investigate the extent to which default activity is

2 modulated by pedagogy, and to shed light on this difference in morphology.

Both early and late response-locked EEG components, ERN and Pe, were observed to vary with age. However, amplitudes for correct and incorrect responses exhibited patterns suggesting that detection and regulation of correct versus incorrect responses may change across development. Correct versus incorrect responses were processed as more different in younger children than older children of our cohort ( $>9$ yo.). Previous work reported stronger behavioral and brain activity for correct rather than incorrect responses in schoolchildren (Denervaud, Fornari, et al., 2020; van den Bos, Guroglu, van den Bulk, Rombouts, \& Crone, 2009). Together these findings suggest a dynamic, likely non-linear, development of error monitoring with possible shift in incorrect versus correct processing of responses across age. It may be that correct responses (less frequent than errors) are first perceived as unexpected early in the learning curve, and not the other way around. This idea could explain inconsistencies reported in developmental studies (Tamnes et al., 2013). Future work should therefore look at correct and incorrect trials separately within a longitudinal framework. Children enrolled in Montessori schools also exhibited a generally larger early brain response, for both correct and incorrect responses. Montessori curricula offer pedagogical tools in the form of self-corrective materials, so that children from early years onwards are trained to explore concepts through trial-and-error strategies, without evaluative feedback such as grades (Marshall, 2017; Montessori, 1936). This approach may reinforce their abilities to monitor response outcomes, independently of correct or incorrect actions. Source estimations for $\triangle \mathrm{ERN}$ confirmed differences in underlying active brain regions. Responses from Montessori and traditionally-schooled children differ in early stages of error monitoring (ERN) within the $\mathrm{cACC}$ and the $\mathrm{mPFC}$. This is in close agreement with previous work on error or conflict-monitoring where the ACC is robustly activated (e.g. 


\section{Running Head: ERROR MONITORING AND BRAIN DEVELOPMENT}

1 Carter et al., 1998; Kiehl, Liddle, \& Hopfinger, 2000; van Veen \& Carter, 2002), and linked

2 to greater prefrontal activity (Botvinick, Cohen, \& Carter, 2004). Furthermore, differences

3 within the left inferior temporal cortex and cerebellum may be related to post-response

4 learning and memorizing processes (Becker et al., 2013; Keren-Happuch, Shen-Hsing, Moon-

5 Ho, \& Desmond, 2014; Marvel \& Desmond, 2010). In the current study, Montessori-schooled

6 children had stronger brain activity for correct rather than incorrect responses, while

7 traditionally-schooled children showed the reverse pattern. It may thus be that early error

8 signals are dissimilar in Montessori and traditional schoolchildren, corroborating recent

9 behavioral and neural findings (Denervaud, Hess, Sander, \& Pourtois, 2020; Denervaud,

10 Fornari, et al., 2020). Future work should continue exploring this idea of a window of

11 opportunity for error monitoring abilities, where experience could have significant impact.

Montessori versus traditionally-schooled children did not differ on

demographic variables, nor on their task-related behavioral outcomes, minimizing

contributions of a selection bias. However, we found some experience-dependent

changes at the neural level, revealing that while subtle changes in fundamental

competences occur, many behavioral measures cannot capture them. This observation

encourages future work in the field of education to combine behavioral and neural

measures, to better capture developmental changes in learning how to learn

(Soderstrom \& Bjork, 2015). To overcome some limitations of the current study,

future work should include a larger sample size, and, optimally, use randomized

21 designs.

Here, we show that while error monitoring and its underlying brain structure, the cACC, undergo major changes in 6- to 12-year-old children, there is a modulatory effect of pedagogy experienced. Given the impact of error monitoring on flexible adaptation later in 
Running Head: ERROR MONITORING AND BRAIN DEVELOPMENT

1 of school pedagogy, beyond behavioral measures, and raises the question of a possible

2 window of plasticity. 
Running Head: ERROR MONITORING AND BRAIN DEVELOPMENT

\section{References}

Aarts, K., De Houwer, J., \& Pourtois, G. (2013). Erroneous and correct actions have a different affective valence: evidence from ERPs. Emotion, 13(5), 960-973. doi:10.1037/a0032808

Beaty, R. E., Benedek, M., Wilkins, R. W., Jauk, E., Fink, A., Silvia, P. J., . . Neubauer, A. C. (2014). Creativity and the default network: A functional connectivity analysis of the

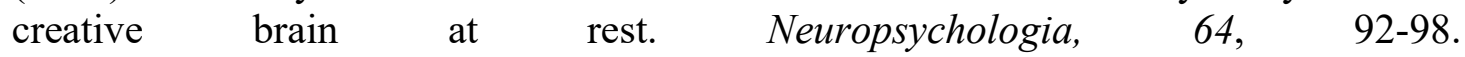
doi:10.1016/j.neuropsychologia.2014.09.019

Blackwell, L. S., Trzesniewski, K. H., \& Dweck, C. S. (2007). Implicit theories of intelligence predict achievement across an adolescent transition: a longitudinal study and an intervention. Child Dev, 78(1), 246-263. doi:10.1111/j.1467-8624.2007.00995.x

Brooker, R. J., Buss, K. A., \& Dennis, T. A. (2011). Error-monitoring brain activity is associated with affective behaviors in young children. Dev Cogn Neurosci, 1(2), 141151. doi:10.1016/j.den.2010.12.002

Carter, C. S., Braver, T., Barch, D. M., Botvinick, M., Noll, D., \& Cohen, J. D. (1998). The role of the anterior cingulate cortex in error detection and the on-line monitoring of performance: An event related fMRI study. Biological Psychiatry, 43, 13s-13s. doi:Doi 10.1016/S0006-3223(98)90491-7

Danielmeier, C., Eichele, T., Forstmann, B. U., Tittgemeyer, M., \& Ullsperger, M. (2011). Posterior medial frontal cortex activity predicts post-error adaptations in task-related visual and motor areas. J Neurosci, 31(5), 1780-1789. doi:10.1523/JNEUROSCI.429910.2011

Denervaud, S., Fornari, E., Yang, X.-F., Hagmann, P., Immordino-Yang, M. H., \& Sander, D. (2020). An fMRI study of error monitoring in Montessori and traditionally-schooled children. npj Science of Learning, 5(1). doi:10.1038/s41539-020-0069-6

Denervaud, S., Hess, A., Sander, D., \& Pourtois, G. (2020). Children's automatic evaluation of self-generated actions is different from adults. Dev Sci, e13045. doi:10.1111/desc.13045

Denervaud, S., Knebel, J. F., Hagmann, P., \& Gentaz, E. (2019). Beyond executive functions, creativity skills benefit academic outcomes: Insights from Montessori education. PLoS One, 14(11), e0225319. doi:10.1371/journal.pone.0225319

Denervaud, S., Knebel, J. F., Immordino-Yang, M. H., \& Hagmann, P. (2020). Effects of Traditional Versus Montessori Schooling on 4- to 15-Year Old children's Performance Monitoring. Mind Brain and Education. doi:https://doi.org/10.1111/mbe.12233

Desikan, R. S., Segonne, F., Fischl, B., Quinn, B. T., Dickerson, B. C., Blacker, D., . . Killiany, R. J. (2006). An automated labeling system for subdividing the human cerebral cortex on MRI scans into gyral based regions of interest. Neuroimage, 31(3), 968-980. doi:10.1016/j.neuroimage.2006.01.021

Elton, M., Band, G., \& Falkenstein, M. (2000). To err is human. Biological Psychology, 51(23), 83-85. doi:Doi 10.1016/S0301-0511(99)00035-6

Falkenstein, M. (2000). Action monitoring, evaluation, and control. Psychophysiology, 37, S9S9.

Falkenstein, M., Hohnsbein, J., Hoormann, J., \& Blanke, L. (1991). Effects of Crossmodal Divided Attention on Late Erp Components .2. Error Processing in Choice Reaction Tasks. Electroencephalography and Clinical Neurophysiology, 78(6), 447-455. doi:Doi 10.1016/0013-4694(91)90062-9 
Fischl, B., Salat, D. H., Busa, E., Albert, M., Dieterich, M., Haselgrove, C., . . . Dale, A. M. (2002). Whole brain segmentation: automated labeling of neuroanatomical structures in the human brain. Neuron, 33(3), 341-355. doi:S089662730200569X [pii]

Fischl, B., Sereno, M. I., \& Dale, A. M. (1999). Cortical surface-based analysis. II: Inflation, flattening, and a surface-based coordinate system. Neuroimage., 9(2), 195-207.

Gennatas, E. D., Avants, B. B., Wolf, D. H., Satterthwaite, T. D., Ruparel, K., Ciric, R., . . . Gur, R. C. (2017). Age-Related Effects and Sex Differences in Gray Matter Density, Volume, Mass, and Cortical Thickness from Childhood to Young Adulthood. $J$ Neurosci, 37(20), 5065-5073. doi:10.1523/JNEUROSCI.3550-16.2017

Genoud, P. (2011). Indice de position socioéconomique (IPSE) : un calcul simplifié:.

Gogtay, N., Giedd, J. N., Lusk, L., Hayashi, K. M., Greenstein, D., Vaituzis, A. C., . . . Thompson, P. M. (2004). Dynamic mapping of human cortical development during childhood through early adulthood. Proc Natl Acad Sci U S A, 101(21), 8174-8179. doi:10.1073/pnas.0402680101

Grammer, J. K., Carrasco, M., Gehring, W. J., \& Morrison, F. J. (2014). Age-related changes in error processing in young children: a school-based investigation. Dev Cogn Neurosci, 9, 93-105. doi:10.1016/j.den.2014.02.001

Hirsh, J. B., \& Inzlicht, M. (2010). Error-related negativity predicts academic performance. Psychophysiology, 47(1), 192-196. doi:10.1111/j.1469-8986.2009.00877.x

Hudson, L. N., Newbold, T., Contu, S., Hill, S. L., Lysenko, I., De Palma, A., . . Purvis, A. (2017). The database of the PREDICTS (Projecting Responses of Ecological Diversity In Changing Terrestrial Systems) project. Ecol Evol, 7(1), 145-188. doi:10.1002/ece3.2579

Immordino-Yang, M. H., Christodoulou, J. A., \& Singh, V. (2012). Rest Is Not Idleness: Implications of the Brain's Default Mode for Human Development and Education. Perspect Psychol Sci, 7(4), 352-364. doi:10.1177/1745691612447308

Kelly, A. M., Di Martino, A., Uddin, L. Q., Shehzad, Z., Gee, D. G., Reiss, P. T., . . Milham, M. P. (2009). Development of anterior cingulate functional connectivity from late childhood to early adulthood. Cereb Cortex, 19(3), 640-657. doi:10.1093/cercor/bhn117

Kiehl, K. A., Liddle, P. F., \& Hopfinger, J. B. (2000). Error processing and the rostral anterior cingulate: An event-related fMRI study. Psychophysiology, 37(2), 216-223. doi:Doi $10.1017 / \mathrm{S} 0048577200990231$

Knebel, J.-F., \& Notter, M. P. (2012). STEN 1.0: Statistical Toolbox for Electrical Neuroimaging (Version 1.0). Zenodo. http://doi.org/10.5281/zenodo.1167723

Leech, R., \& Sharp, D. J. (2014). The role of the posterior cingulate cortex in cognition and disease. Brain, 137, 12-32. doi:10.1093/brain/awt162

Lillard, A., \& Else-Quest, N. (2006). The early years. Evaluating Montessori education. Science, 313(5795), 1893-1894. doi:10.1126/science.1132362

Lillard, A. S. (2005). Montessori : the science behind the genius. New York: Oxford University Press.

Lillard, A. S. (2011). Mindfulness Practices in Education: Montessori's Approach. Mindfulness, 2(2), 78-85. doi:10.1007/s12671-011-0045-6

Margulies, D. S., Kelly, A. M., Uddin, L. Q., Biswal, B. B., Castellanos, F. X., \& Milham, M. P. (2007). Mapping the functional connectivity of anterior cingulate cortex. Neuroimage, 37(2), 579-588. doi:10.1016/j.neuroimage.2007.05.019

Marshall, C. (2017). Montessori education: a review of the evidence base. npj Science of Learning, 2(1). doi:10.1038/s41539-017-0012-7 
Menon, V., \& Uddin, L. Q. (2010). Saliency, switching, attention and control: a network model of insula function. Brain Structure \& Function, 214(5-6), 655-667. doi:10.1007/s00429010-0262-0

Meyer, A., Weinberg, A., Klein, D. N., \& Hajcak, G. (2012). The development of the errorrelated negativity (ERN) and its relationship with anxiety: evidence from 8 to 13 yearolds. Dev Cogn Neurosci, 2(1), 152-161. doi:10.1016/j.dcn.2011.09.005

Montessori, M. (1936). The Secret of Childhood (1981 ed.). New York: Ballantine.

Murphy, P. R., van Moort, M. L., \& Nieuwenhuis, S. (2016). The Pupillary Orienting Response Predicts Adaptive Behavioral Adjustment after Errors. PLoS One, 11(3), e0151763. doi:10.1371/journal.pone.0151763

Nieuwenhuis, S., Ridderinkhof, K. R., Blom, J., Band, G. P., \& Kok, A. (2001). Error-related brain potentials are differentially related to awareness of response errors: evidence from an antisaccade task. Psychophysiology, 38(5), 752-760.

Notebaert, W., Houtman, F., Opstal, F. V., Gevers, W., Fias, W., \& Verguts, T. (2009). Posterror slowing: an orienting account. Cognition, 111(2), 275-279. doi:10.1016/j.cognition.2009.02.002

Overbeek, T. J. M., Nieuwenhuis, S., \& Ridderinkhof, K. R. (2005). Dissociable components of error processing - On the functional significance of the Pe Vis-a-vis the ERN/Ne. Journal of Psychophysiology, 19(4), 319-329. doi:10.1027/0269-8803.19.4.319

Peirce, J., Gray, J. R., Simpson, S., MacAskill, M., Hochenberger, R., Sogo, H., . . Lindelov, J. K. (2019). PsychoPy2: Experiments in behavior made easy. Behav Res Methods. doi:10.3758/s13428-018-01193-y

Posner, M. I., \& Rothbart, M. K. (2007). Educating the human brain (1st ed.). Washington, DC: American Psychological Association.

Posner, M. I., Rothbart, M. K., Sheese, B. E., \& Tang, Y. (2007). The anterior cingulate gyrus and the mechanism of self-regulation. Cogn Affect Behav Neurosci, 7(4), 391-395.

Raichle, M. E., MacLeod, A. M., Snyder, A. Z., Powers, W. J., Gusnard, D. A., \& Shulman, G. L. (2001). A default mode of brain function. Proc Natl Acad Sci U S A, 98(2), 676-682. doi:10.1073/pnas.98.2.676

Rathunde, K. (2001). Montessori Education and optimal experieunce: a framework for new research. The NAMTA Journal, 26(1), 11-43.

Raven, J., Raven, J. C., \& Court, J. H. (2003). Manual for Raven's Progressive Matrices and Vocabulary Scales. Section 1: General Overview. . San Antonio, TX: Harcourt Assessment.

Smulders, S. F., Soetens, E., \& van der Molen, M. W. (2016). What happens when children encounter an error? Brain Cogn, 104, 34-47. doi:10.1016/j.bandc.2016.02.004

Snodgrass, J. G., \& Vanderwart, M. (1980). A standardized set of 260 pictures: norms for name agreement, image agreement, familiarity, and visual complexity. J Exp Psychol Hum Learn, 6(2), 174-215. doi:10.1037//0278-7393.6.2.174

Soderstrom, N. C., \& Bjork, R. A. (2015). Learning versus performance: an integrative review. Perspect Psychol Sci, 10(2), 176-199. doi:10.1177/1745691615569000

Spielberger, C. D., \& Vagg, P. R. (1984). Psychometric properties of the STAI: a reply to Ramanaiah, Franzen, and Schill. $J$ Pers Assess, 48(1), 95-97. doi:10.1207/s15327752jpa4801_16

Sunil, M., Hieu, H. Q., Arjan Singh, R. S., Ponnampalavanar, S., Siew, K. S. W., \& Loch, A. (2019). Evolving trends in infective endocarditis in a developing country: a consequence of medical progress? Ann Clin Microbiol Antimicrob, 18(1), 43. doi:10.1186/s12941-019-0341-X 
Talairach, J., \& Tournoux, P. (1988). Co-planar stereotaxic atlas of the human brain : 3dimensional proportional system : an approach to cerebral imaging. Stuttgart; New York: Georg Thieme.

Tamnes, C. K., Walhovd, K. B., Torstveit, M., Sells, V. T., \& Fjell, A. M. (2013). Performance monitoring in children and adolescents: a review of developmental changes in the errorrelated negativity and brain maturation. Dev Cogn Neurosci, 6, 1-13. doi:10.1016/j.den.2013.05.001

Uddin, L. Q., Supekar, K. S., Ryali, S., \& Menon, V. (2011). Dynamic reconfiguration of structural and functional connectivity across core neurocognitive brain networks with development. $J$ Neurosci, 31(50), 18578-18589. doi:10.1523/JNEUROSCI.446511.2011

Ullsperger, M., \& Danielmeier, C. (2016). Reducing Speed and Sight: How Adaptive Is PostError Slowing? Neuron, 89(3), 430-432. doi:10.1016/j.neuron.2016.01.035

Ullsperger, M., Danielmeier, C., \& Jocham, G. (2014). Neurophysiology of performance monitoring and adaptive behavior. Physiol Rev, 94(1), 35-79. doi:10.1152/physrev.00041.2012

van den Bos, W., Guroglu, B., van den Bulk, B. G., Rombouts, S. A. R. B., \& Crone, E. A. (2009). Better than expected or as bad as you thought? The neurocognitive development of probabilistic feedback processing. Frontiers in Human Neuroscience, 3. doi:ARTN 52

10.3389/neuro.09.052.2009

van Veen, V., \& Carter, C. S. (2002). The anterior cingulate as a conflict monitor: fMRI and ERP studies. Physiol Behav, 77(4-5), 477-482.

Velanova, K., Wheeler, M. E., \& Luna, B. (2008). Maturational Changes in Anterior Cingulate and Frontoparietal Recruitment Support the Development of Error Processing and Inhibitory Control. Cerebral Cortex, 18(11), 2505-2522. doi:10.1093/cercor/bhn012 
4

Independent Samples T-Test

\begin{tabular}{lllrll}
\hline \multicolumn{2}{c}{ Mean (SD) } & & & \\
& Montessori & Traditional & Statistic & $\boldsymbol{p}$ & Cohen's d \\
Age & $8.82(1.64)$ & $8.74(1.65)$ & 0.04 & 0.97 & 0.02 \\
PM-47 & $34.08(2.35)$ & $32.36(3.27)$ & 1.52 & 0.14 & 0.60 \\
Anxiety & $13.67(6.39)$ & $13.14(4.88)$ & 0.24 & 0.82 & 0.09 \\
Mindset & $13.46(6.19)$ & $15.62(4.99)$ & -1.54 & 0.14 & 0.63 \\
SSE & $7.18(0.87)$ & $7.50(1.24)$ & -0.72 & 0.48 & 0.29 \\
Parental Style (score) & $26.82(4.53)$ & $26.86(4.15)$ & -0.02 & 0.98 & 0.01
\end{tabular}


A. Cortical changes from 6 to 12 yo.

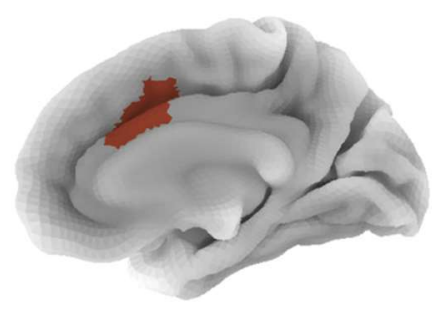

C. $\quad \mathrm{ACC}$ and ERN relationship

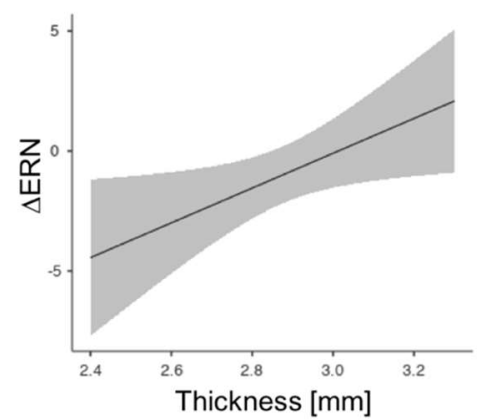

B. Error-Related Negativity (ERN)

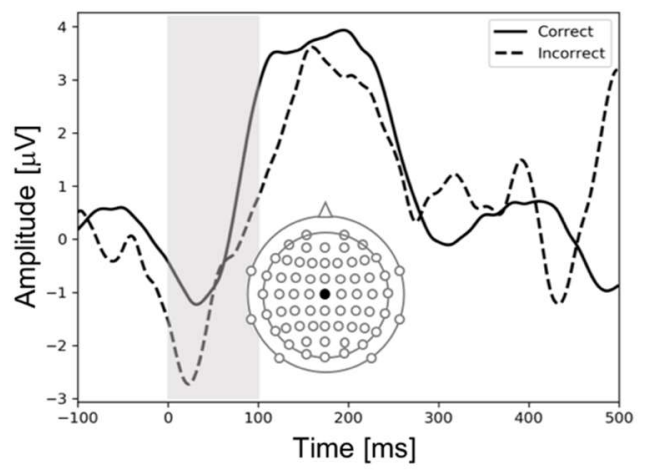

D. Modulation by school experience (ERN)

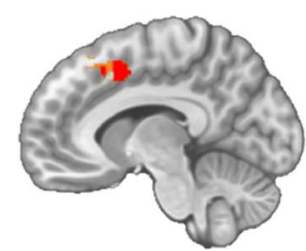

Source estimation, $\alpha<0.05$ 